TITLE:

\title{
Time-resolved imaging of "Plasma Bullets" in a dielectric capillary atmospheric pressure discharge
}

\section{$\operatorname{AUTHOR}(\mathrm{S}):$}

Sands, Brian L.; Ganguly, Biswa N.; Tachibana, Kunihide

\section{CITATION:}

Sands, Brian L. ... [et al]. Time-resolved imaging of "Plasma Bullets" in a dielectric capillary atmospheric pressure discharge. IEEE TRANSACTIONS ON PLASMA SCIENCE 2008, 36(4(Part 1)): 956-957

\section{ISSUE DATE:}

2008-08

URL:

http://hdl.handle.net/2433/84552

\section{RIGHT:}

(c) 2008 IEEE. Personal use of this material is permitted. However, permission to reprint/republish this material for advertising or promotional purposes or for creating new collective works for resale or redistribution to servers or lists, or to reuse any copyrighted component of this work in other works must be obtained from the IEEE. 


\title{
Time-Resolved Imaging of "Plasma Bullets" in a Dielectric Capillary Atmospheric Pressure Discharge
}

\author{
Brian L. Sands, Biswa N. Ganguly, and Kunihide Tachibana, Member, IEEE
}

\begin{abstract}
Temporally resolved images acquired using a 5-ns gated intensified charge-coupled device camera show the dynamical behavior of a plasma jet emanating from the end of a dielectric capillary operating at atmospheric pressure. Broadband emission and filtered emission from excited $\mathrm{Ar}, \mathrm{He}, \mathrm{N}_{2}$ and $\mathrm{N}_{2}^{+}$species were acquired. The properties of the highly localized so-called "plasma bullets" that make up the plasma jet strongly resemble the properties of cathode-directed streamers in positive corona discharges.
\end{abstract}

Index Terms-Atmospheric pressure plasma jet (APPJ), dielectric capillary, streamers, time-resolved optical imaging.

$\mathbf{S}$ EVERAL atmospheric pressure plasma jet (APPJ) configurations are actively being studied for a variety of applications. These include single-cell configurations and multicell arrays. The single-cell dielectric capillary configuration presented here, with cylindrical, coaxial but nonconcentric electrodes, was introduced by Teschke et al. [1] and has been studied in various forms [2], [3]. This configuration is characterized by a capillary dielectric barrier discharge (CDBD) created within the gap between the electrodes and a plasma jet extending beyond the capillary tip into the open air.

We used a cylindrical glass capillary with a 2-mm inner diameter (ID) and a 3-mm outer diameter (OD). The copper ring electrodes surround the capillary OD and were spaced $1 \mathrm{~cm}$ apart. The anode was placed close to the edge of the capillary. The electrodes were energized with a unipolar voltage pulse with a rise time of $20 \mathrm{~ns}$. A gated intensified chargecoupled device (ICCD) camera, with a 5-ns gate width, was synchronized to these voltage pulses to acquire the images in Fig. 1.

Fig. 1 shows a set of four emission sequences from the discharge, each showing eight consecutively acquired ICCD images at $10 \mathrm{~ns}$ intervals from the onset of emission. A magnified true-color photograph of the plasma jet and CDBD is shown in Fig. 1(a) for reference. The discharge was excited using an $11-\mathrm{kV}$ voltage pulse at a $1 \mathrm{kHz}$ repetition rate with a 5\% $\mathrm{Ar} / \mathrm{He}$ mixture flowing through the capillary at a rate of $\sim 4.5$ standard liters per minute. Fig. 1(b)-(e) respectively depict unfiltered broadband emission, $750 \mathrm{~nm}$ emission from the

Manuscript received November 2, 2007; revised January 7, 2008.

B. L. Sands is with the UES, Inc., Dayton, OH 45433 USA (e-mail: brian.sands@wpafb.af.mil).

B. N. Ganguly is with the Air Force Research Laboratory, Dayton, OH 45433 USA (e-mail:biswa.ganguly@wpafb.af.mil).

K. Tachibana is with the Department of Electronic Science and Engineering, Kyoto University, Kyoto 615-8510, Japan.

Digital Object Identifier 10.1109/TPS.2008.917789
Ar $3 \mathrm{p}^{5} 4 \mathrm{p}-3 \mathrm{p}^{5} 4 \mathrm{~s}$ transition, both $391 \mathrm{~nm}$ emission from $\mathrm{N}_{2}^{+} \mathrm{B}^{2} \Sigma_{u}-\mathrm{X}^{2} \Sigma_{g}(0,0)$ and $389 \mathrm{~nm}$ emission from the $\mathrm{He}$ $3^{3} \mathrm{P}-2^{3} \mathrm{~S}_{1}$ transition, and $337 \mathrm{~nm}$ emission from $\mathrm{N}_{2} \mathrm{C}^{3} \Pi_{u}-$ $\mathrm{B}^{3} \Pi_{g}(0,0)$. An 8-bit false-color intensity map was applied to each of the sequence frames in Fig. 1(b)-(e). Normalized intensity scales are shown in the figure for reference and apply only to the specified subfigure. There is actually a large variation in intensity when comparing between the subfigures.

The most striking feature in Fig. 1 is that what appears to be a continuous jet to the eye [Fig. 1(a)] is actually a highly localized, propagating region of emission when viewed on a time-resolved scale. Each slice from the four image sequences shown was integrated over a period that ranged from 100 pulses for the unfiltered and $750 \mathrm{~nm}$ emission to 600 pulses for the weaker nitrogen emission bands to acquire sufficient signal to noise. This indicates a high degree of shot to shot repeatability as there is very little streaking apparent in the images. The time-resolved emission from this dielectric capillary configuration was first acquired by Teschke et al. [1] who termed the propagating ionization front as a "plasma bullet." The "plasma bullet" was only visible following the rising edge of the voltage pulse. The choice of filters revealed the structure of the "plasma bullet." Examination of Fig. 1(b) and (c) shows that the "plasma bullet" is not homogeneous, but is composed of a distinct region of emission from the $\mathrm{Ar} / \mathrm{He}$ flow-gas plasma bounded by the capillary ID that is surrounded by nitrogen emission from an air plasma envelope. These are basic properties of the "plasma bullet" that have been observed previously [1]. The filtered emission covering the $389 \mathrm{~nm} \mathrm{He}$ and $391 \mathrm{~nm} \mathrm{~N}_{2}^{+}$transitions [Fig. 1(d)] tracks the ionization front and clearly outlines the "plasma bullet" while emission from excited $\mathrm{N}_{2}$ at $337 \mathrm{~nm}$ [Fig. 1(e)] shows a weakly ionized plasma channel that persists behind the propagating front.

Noting the length scale indicated in the figure, the "plasma bullet" traverses about $3.5 \mathrm{~cm}$ in $70 \mathrm{~ns}$. Such high propagation velocities on the order of $10^{7} \mathrm{~cm} / \mathrm{s}$ have been consistently observed [1], [3]. A closer examination of the figure shows propagation with a constant velocity over much of the range with a visible deceleration noted in the final stages of the "plasma bullet." Perhaps the most important feature to note in Fig. 1 is the relative timing of the plasma jet and inner CDBD. The plasma jet is initiated $\gtrsim 10 \mathrm{~ns}$ before the CDBD inside is initiated. This has not previously been observed and indicates that the plasma jet is a self-sustained discharge independent from the interior CDBD.

Using a different electrode arrangement, Lu and Laroussi [3] observed similarly high propagation velocities and suggested 


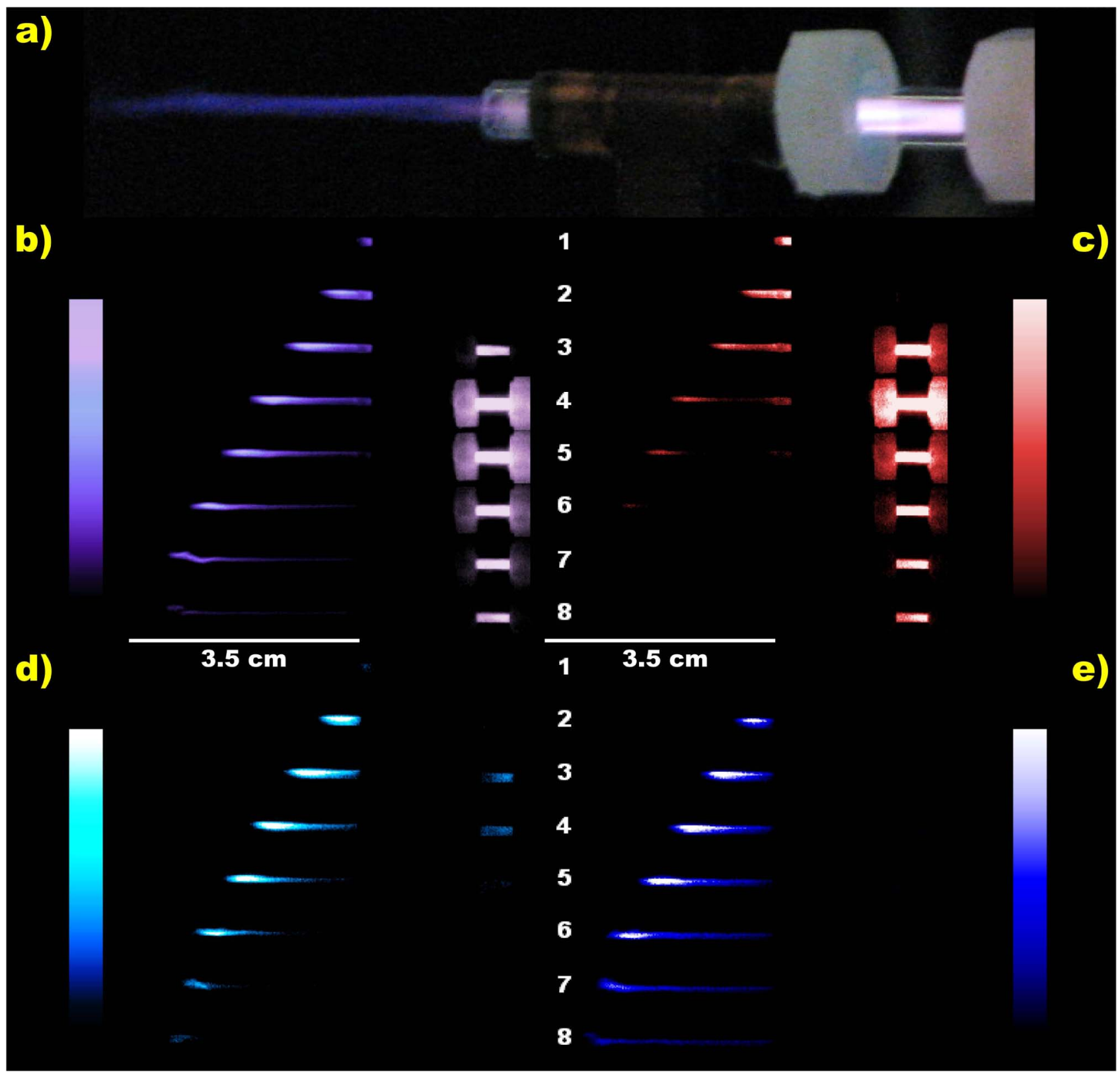

Fig. 1. (a) Magnified true-color photograph of the dielectric capillary APPJ. (b) False-color intensity maps representing time-resolved broadband emission, (c) excited Ar emission lines near $750 \mathrm{~nm}$, (d) emission from $\mathrm{N}_{2}^{+}$at 391 and $389 \mathrm{~nm}$ emission from excited He, and (e) excited $\mathrm{N}_{2}$ emission at $337 \mathrm{~nm}$ were acquired using an ICCD camera gated at $5 \mathrm{~ns}$. Each frame represents an increment of $10 \mathrm{~ns}$.

a streamer discharge mechanism similar to positive corona streamers. Our observations, gleaned from Fig. 1 and confirmed using more quantitative time-resolved spectroscopic measurements, strongly support this interpretation. Experimental observations and theoretical modeling of streamer discharge dynamics in point-plane positive corona setups show similar features to those observed in our configuration. There are some differences, however. Positive corona streamers can trace multiple paths with sometimes several bifurcations along the way and are not as repeatable in pulsed setups. The streamers associated with this dielectric capillary configuration are, in contrast, very repeatable and highly directed, tracing the same path consistently. We believe this can partly be attributed to the accumulation of charge on the capillary tip and the focusing of the electric field along the flow axis.

\section{REFERENCES}

[1] M. Teschke, J. Kedzierski, E. G. Finantu-Dinu, D. Korzec, and J. Engemann, "High-speed photographs of a dielectric barrier atmospheric pressure plasma jet," IEEE Trans. Plasma Sci., vol. 33, no. 2, pp. 310-311, Apr. 2005

[2] O. Sakai, Y. Kishimoto, and K. Tachibana, "Integrated coaxial-hollow micro dielectric-barrier-discharges for a large-area plasma source operating at around atmospheric pressure," J. Phys. D: Appl. Phys., vol. 38, no. 3, pp. 431-441, Feb. 2005.

[3] X. Lu and M. Laroussi, "Dynamics of an atmospheric pressure plasma plume generated by submicrosecond voltage pulses," J. Appl. Phys., vol. 100, no. 6, p. 063302 , Sep. 2006 